Committee, the Research \& Development Committee and the MC-FP Committee.

Howard Blanchette, MD is Professor and Chairman of Obstetrics and Gynecology at New York Medical College. He is also the Director of the Department of Obstetrics and Gynecology at Westchester Medical Center. In addition, Dr. Blanchette serves as a member of the Board of Directors of the American Board of Obstetrics and Gynecology. Dr. Blanchette will serve the ABFM on the Information \& Technology Committee and the Communications/Publications Committee.

The remaining current members of the Board are: Arlene Brown, MD of Ruidoso, New Mexico; Thomas H. Cogbill, MD of LaCrosse, Wisconsin, Susan C. Day, MD of Philadelphia, Pennsylvania; Thomas P. Gessner, MD of Latrobe, Pennsylvania; Samuel Jones, MD of Fairfax, VA, Warren P. Newton, MD of Chapel Hill, North Carolina; Daniel K. Winstead, MD of New Orleans, Louisiana; and Michael G. Workings, MD of Detroit, Michigan.

Jane Ireland

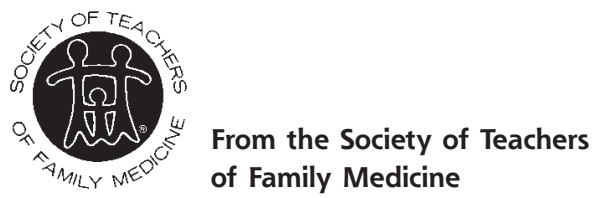

Ann Fam Med 2009;7:469-470. doi:10.1370/afm.1049.

\section{STFM AND THE STFM FOUNDATION ANNOUNCE THE 2009 GROUP PROJECT FUND WINNERS}

The STFM Group Project Fund, supported by the STFM Foundation, was established to promote and support innovative educational projects proposed by STFM groups.

This fund encourages STFM group members to collaboratively plan, develop, implement, evaluate, and disseminate findings from educationally related scholarly projects that benefit group members, STFM, and the discipline of family medicine. STFM Foundation sets aside $50 \%$ of the undesignated net proceeds of each annual giving campaign to fund these projects.

\section{The 2009 Group Project Fund Winners}

PROJECT: Online Training in Dietary Supplements for Family Physicians

Many patients use dietary supplements, and physicians need evidence-based, up-to-date information on the safety and efficacy of dietary supplements. This project will develop, evaluate, and market online continuing medical education modules on dietary supplements and 3 chronic health conditions: heart disease, diabetes, and asthma. These modules will be maintained and updated at the Boston Medical Center Department of Family Medicine and housed on the Boston University (BU) Distance Education for Health Web site. CME credit will be through BU.

STFM Group: Integrative Medicine-Paula Gardiner, MD, MPH, Boston University Department of Family Medicine, principal investigator

Award: \$9,745 over 2 years

PROJECT: Medical School Admission Policies and the Family Medicine Pipeline: Developing Practical Guidance Based on Analysis of Student Origins Many state medical schools have increased their efforts to recruit and matriculate out-of-state medical students in the past decade, while they simultaneously have experienced decreased numbers of students matriculating into the specialty of family medicine. Practical information is lacking on the relationship between student origin and medical specialty choice. This project will address this deficiency by analyzing data from the 10-year period prior to 1997, which saw a growth in the number of US seniors entering family medicine, as well as the 10-year period after 1997 in which a decline occurred. The project is designed to alleviate potential unintended consequences of current policies of admissions committees and to empower administrators to make informed decisions that will benefit underserved rural populations by increasing the recruitment of physicians from rural areas.

STFM Group: Rural Health-Richard Pretorius, MD, MPH, SUNY Department of Family Medicine, Buffalo, NY, principal investigator

Award: $\$ 10,000$ over 2 years

PROJECT: Current Trends in Medical Education in Identifying and Treating Patients Exposed to Domestic Violence

Residency Review Committee (RRC) guidelines state that each residency must have a structured curriculum in community medicine, including didactic and experiential components for the assessment of risks for abuse, neglect, and family and community violence along with a process to evaluate the curriculum and to document resident skill attainment. This project will survey family medicine departments with regard to the training of faculty, residents, and medical students in the assessment of these risks within the framework of ACGME and RRC guidelines. The results will characterize the field's current focus, identifying systematic 
educational gaps in addition to deconstructing the components of innovative models that can be disseminated nationally.

STFM Group: Violence Education and Prevention-Peter Cronbolm, MD, MSCE, University of Pennsylvania Department of Family Medicine and Community Health, principal investigator

Award: $\$ 4,000$ for 1 year

Requests for proposals are sent annually via e-mail to STFM group chairs in September. Any recognized, active STFM Group may submit a proposal for funding. Proposals must be received in the STFM office by December 1 .

In the 2009 funding cycle, the STFM Foundation was unable to fund several projects of merit due to limited funds. For more information about the STFM Group Project Fund or to donate to the STFM Foundation to help support projects like those listed above, visit http://www.stfm.org/fnd.

Traci Nolte, CAE, Communications Director Society of Teachers of Family Medicine

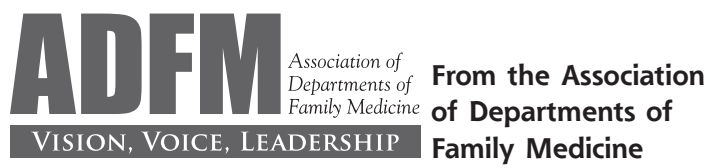

Ann Fam Med 2009;7:470-471. doi:10.1370/afm.1046.

\section{IMPACT OF EXPANDING USE OF HEALTH INFORMATION TECHNOLOGIES ON MEDICAL STUDENT EDUCATION IN FAMILY MEDICINE}

The call for increased health care quality and access has facilitated the expanded use of health information technology (HIT) in the United States. In hospitals and physicians' offices, HIT is most likely represented by the electronic health record (EHR) which, when fully deployed, provides healthcare information storage, results management, medical decision support, order entry functions, and multiple portals for provider and patient access. HIT has not yet been widely adopted in physician offices, and data are not yet available to document hoped-for benefits of improved care and lower cost. HIT needs continuing study to determine how to most effectively use these technologies to achieve the intended improvement in health care quality. The incomplete adoption and deployment of EHRs present unique opportunities and challenges for medical student educa- tion in FM which are elucidated in this commentary.

The adoption of EHRs in hospitals and physicians' offices has been slower than anticipated based in large part on purchase and maintenance costs as well as the workforce behavioral changes and office workflow changes required for optimum implementation. The inconsistent deployment of components of the EHR creates wide variability of functions between different clinical venues. This variability is more likely in ambulatory/office settings where the degree of deployment and implementation is based on practice size, local decisions, and tolerances as opposed to hospitals where regulatory and fiscal forces more likely prevail. In community private practice offices of 1 to 5 physicians, general EHR adoption rates, which is directly related to practice size, range from $16 \%$ to $25 \%$ and EHRs with core functions fully implemented range from $4.4 \%$ to $10.2 \%$.

FM faculty members' participation in introductory clinical courses provides opportunities to prepare students for effective learning in clinical settings supported by EHRs and other components of HIT. These courses should emphasize the effective use of EHRs to facilitate information synthesis and interpretation, clinical decision making, and patient doctor communication. Unfortunately, many introductory courses contain mostly product-specific technical details with less emphasis on the use of EHRs to promote learning in the context of patient centered care.

These introductory EHR courses may not adequately prepare students for FM Clerkship experiences in multiple teaching sites with different rates of EHR adoption and implementation. Students with expectations of health care systems with heavy integration of HIT may view FM Clerkships sites as not uniformly representing contemporary medical practice. The IT competency of FM faculty both in community and academic settings may vary greatly, making the educational use of EHRs inconsistent. This lack of consistency adds another layer of complexity to faculty development needs, especially for those teaching programs using multiple and widely distributed community settings.

The effects of EHRs on medical student learning is not known. To date, small pilot studies have only compared reported use of EHR tools and student attitudes. There is no research evaluating the impact of EHRs on medical student learning. Assessing impact of EHRs on learning in FM Clerkships is made more difficult because of the varied ambulatory clinical venues and most do not have a competency-based curriculum with measureable educational outcomes. The ultimate impact of HIT and the use of EHRs on student learning requires scholarly inquiry by the FM medical student education community to ensure the tools are 